\title{
Mg impurity in helium droplets
}

\author{
J. Navarro \\ IFIC (CSIC-Universidad de Valencia), \\ Apartado Postal 22085, E-46.071-Valencia, Spain \\ D. Mateo and M. Barranco \\ Departament E.C.M., Facultat de Física, and $I N^{2} U B$, \\ Universitat de Barcelona. Diagonal 647, E-08028 Barcelona, Spain \\ A. Sarsa \\ Departamento de Física, Facultad de Ciencias, \\ Universidad de Córdoba. E-14071 Córdoba, Spain
}

(Dated: April 25, 2018)

\begin{abstract}
Within the diffusion Monte Carlo approach, we have determined the structure of isotopically pure and mixed helium droplets doped with one magnesium atom. For pure ${ }^{4}$ He clusters, our results confirm those of M. Mella et al. [J. Chem. Phys. 123, 054328 (2005)] that the impurity experiences a transition from a surface to a bulk location as the number of helium atoms in the droplet increases. Contrarily, for pure ${ }^{3} \mathrm{He}$ clusters $\mathrm{Mg}$ resides in the bulk of the droplet due to the smaller surface tension of this isotope. Results for mixed droplets are presented. We have also obtained the absorption spectrum of $\mathrm{Mg}$ around the $3 s 3 p^{1} P_{1} \leftarrow 3 s^{2}{ }^{1} S_{0}$ transition.
\end{abstract}

PACS numbers: 36.40.-c, 33.20.Kf, 67.60.gj 


\section{INTRODUCTION}

The solubility of alkaline earth atoms attached to helium droplets has been found to critically depend on the dopant and on the helium isotope droplets are made of. This is at clear variance with what happens for alkali atoms, that are all found to reside in a dimple at the surface of the droplet irrespective of their isotopic composition,,$\frac{1,2}{2}$ or to impurities that experience a large attractive interaction with helium as, e.g., inert gas atoms that are found to reside in the bulk of the droplet. This is a particular yet prominent aspect of a much broader subject -the physics and chemistry of pristine and doped helium nanodroplets- that has been reviewed in a series of articles, see e.g. Refs. $3-8$ and references therein.

Ancilotto and coworkers ${ }^{9}$ have provided a solvation criterion for impurities in liquid helium in terms of the dimensionless parameter

$$
\lambda=\frac{\rho \varepsilon r_{e}}{2^{1 / 6} \sigma}
$$

where $\rho$ and $\sigma$ are the density and the surface tension of liquid He, respectively, and $\varepsilon$ and $r_{e}$ are the well depth and the equilibrium distance of the He-impurity interaction, respectively. This parameter measures the balance between the energy of the impurity and the surface energy of the liquid. If $\lambda<1.9$, the impurity sits on the free surface of the fluid and no solvation occurs. For $\mathrm{Mg}$ one has $\lambda=2.6$ for ${ }^{4} \mathrm{He}$ and $\lambda=4.6$ for ${ }^{3} \mathrm{He}$. Since this criterion does take into account neither the complexities of the system nor the fine details of the He-impurity pair potential and it has been established for bulk liquid, it must be taken with care when the value of $\lambda$ is fairly close to 1.9, in which case only a detailed calculation may unveil the solvation properties of a given impurity in He drops.

It has been found experimental and theoretically that $\mathrm{Ca}$ atoms solvate in ${ }^{3} \mathrm{He}$ but not in ${ }^{4} \mathrm{He}$ droplets, and that depending on their isotopic composition, they may reside in the ${ }^{3} \mathrm{He}-{ }^{4} \mathrm{He}$ interface that develops in mixed droplets at low temperatures $\underline{10} \underline{-12}$ The lighter alkaline earth Mg presents a borderline behavior. Within Density Functional Theory (DFT), it has been found to solvate in ${ }^{3} \mathrm{He}$ droplets and to be very delocalized in ${ }^{4} \mathrm{He}$ ones. ${ }^{10,13}$ Previous diffusion Monte Carlo calculations (DMC) ${ }^{14}$ have yielded the result that $\mathrm{Mg}$ is fully solvated for a critical number of ${ }^{4} \mathrm{He}$ atoms of about $N_{4}=30$. A similar transition from

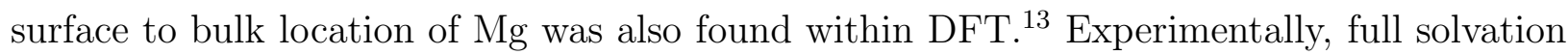
of $\mathrm{Mg}$ in ${ }^{4} \mathrm{He}$ droplets has been inferred from the analysis of Laser Induced Fluorescence (LIF) $\stackrel{15}{\frac{15}{2}}$ comparing it with LIF experiments on the absorption spectrum of $\mathrm{Mg}$ atoms in 
liquid ${ }^{4}$ He. ${ }^{16,17}$ More recent Resonant Two-Photon-Ionization (R2PI) experiments $\frac{18}{}$ have also yielded a bulk location for this impurity.

Since an electron-impact ionization experiment $\frac{19}{}$ carried out in $\mathrm{Mg}$ doped ${ }^{4} \mathrm{He}$ drops with about $10^{4}$ atoms seems to indicate that magnesium reside at or near the droplet surface, and some unpublished DMC calculations seem also to point toward a rather surface location of $\mathrm{Mg}$ in ${ }^{4} \mathrm{He}$ droplets, ${ }^{20-22}$ we have undertaken an independent Monte Carlo analysis of the structure of these systems aiming at settling this issue, complementing the previous analyses with a DMC study of ${ }^{3} \mathrm{He}$ and mixed ${ }^{3} \mathrm{He}-{ }^{4} \mathrm{He}$ droplets doped with $\mathrm{Mg}$ that has not been previously carried out. The interest in addressing doped mixed helium clusters has been already stressed. $\stackrel{12}{\underline{12}}$ Very recently, we have studied the structure and absorption spectrum of $\mathrm{Mg}$ in liquid helium mixtures as a function of pressure and isotopic composition. ${ }^{2}$

This paper is organized as follows. In Sec. II we give some details about the DMC calculations. In Sec. III we present our results, and in Sec. IV we present a brief summary.

\section{METHODS}

Quantum Monte Carlo methods aim at solving the Schrödinger equation of a many-body system with the only knowledge of the inter-particle interaction. In our calculations we have employed the He-He Aziz potential, ${ }^{23}$ and the $X^{1} \Sigma \mathrm{Mg}$-He interaction as obtained by Hinde. ${ }^{24}$ For the description of the absorption spectrum described in Sec IIC, the $\Sigma$ and $\Pi$ Mg-He pair potentials of Ref. 14 have been used. To facilitate the use of these potentials, we have fitted them (by simulated annealing) to an analytical expression of the kind

$$
\begin{gathered}
V_{M g-H e}(r)=A e^{-\alpha r-\beta r^{2}}-F(r) \times\left(\frac{C_{6}}{r^{6}}+\frac{C_{8}}{r^{8}}+\frac{C_{10}}{r^{10}}\right) \\
F(r)= \begin{cases}e^{-(1-D / r)^{2}} & r<D \\
1 & r \geq D\end{cases}
\end{gathered}
$$

The parameters are given in Table I. The resulting fits are shown in Fig. 1, together with the calculated values (indicated with symbols in the figure). It can be seen that the agreement is excellent. For the sake of comparison, we have also included the He-He interaction used in this work.

Our DMC calculations are based on a variational or importance sampling wave function. In that case, one does not solve the Schrödinger equation for the true wave function $\Psi(\mathcal{R}, t)$, 
but for the auxiliary function $f(\mathcal{R}, t)=\Psi_{T}(\mathcal{R}) \Psi(\mathcal{R}, t)$. The trial wave function $\Psi_{T}(\mathcal{R})$ guides the random walk and minimizes the variance. We have used a rather simple form, containing the basic required properties, the same as employed in the past to describe a $\mathrm{Ca}$ impurity. $\underline{\underline{12}}$ It is a generalization of the trial function adopted in previous studies on pristine mixed helium clusters,$\underset{25,26}{2}$ and is written as a product of seven terms

$$
\Psi_{T}(\mathcal{R})=\Psi_{44} \Psi_{33} \Psi_{34} \Psi_{4 \mathrm{Mg}} \Psi_{3 \mathrm{Mg}} D_{\uparrow} D_{\downarrow},
$$

where $\{\mathcal{R}\}$ represents the set of $3\left(N_{3}+N_{4}+1\right)$ coordinates of the atoms forming the cluster, $N_{3}\left(N_{4}\right)$ being the number of ${ }^{3} \mathrm{He}\left({ }^{4} \mathrm{He}\right)$ atoms. The first five terms are Jastrow factors $\Psi_{M N}$ for each pair $(M, N)$ of different atoms, for which we have chosen the generic form

$$
\Psi_{M N}=\prod_{i \neq j} \exp \left(-\frac{1}{2}\left[\frac{b_{M N}}{r_{i j}}\right]^{\nu_{M N}}-\alpha_{M N} r_{i j}\right),
$$

where indices $i, j$ run over the corresponding type of atom, and includes a short-range repulsion term with parameters $b_{M N}$ and $\nu_{M N}$ associated with it, and a long-range confining term with corresponding parameter $\alpha_{M N}$. The form of the short-range repulsion term was introduced long ago by McMillan $\frac{27}{-}$ to describe the homogeneous liquid ${ }^{4} \mathrm{He}$ using a 12-6 LennardJones interaction. In that case, the values of the two parameters $\nu$ and $b$ are fixed by the short-range behavior of a pair of atoms, and one gets $\nu=5$, and $b=\left[16 \mu \epsilon /\left(25 \hbar^{2}\right)\right]^{1 / 10} \sigma^{6 / 5}$, where $\mu$ is the reduced mass for each pair of atoms, and $\epsilon$ and $\sigma$ are the Lennard-Jones energy and distance parameters, respectively.

Notice that each $\Psi_{M N}$ function is explicitly symmetric under the exchange of identical particles. The antisymmetry required for ${ }^{3} \mathrm{He}$ fermions is incorporated in the Slater determinants $D_{\uparrow}$ and $D_{\downarrow}$, related to the spin-up and -down fermions, respectively. Two aspects should be considered in these determinants, namely the form and the filling of the single particle orbitals. As in previous works $\underline{25}, 26$ the determinants have been built up with homogeneous monomials of the fermion Cartesian coordinates as $x_{i}^{n_{x}} y_{i}^{n_{y}} z_{i}^{n_{z}}$, where the subindex $i$ refers to the particle, and the integer number $n=n_{x}+n_{y}+n_{z}$ defines a "shell". This kind of Slater determinant has also been employed for other many-body systems, (see e.g. Ref. 28,29). It turns out that they are of Vandermonde type and, provided that the shells are filled in an increasing order of $n$, they can be expressed in terms of products of the relative coordinates, being thus translationally invariant. $\underline{28,29}$ Besides, we have always assumed a filling scheme in which the total spin is minimum, either 0 or $1 / 2$, for $N_{3}$ even or odd, re- 
spectively. For some pure ${ }^{3}$ He clusters, the shell filling has been given in Ref. 26, indicating the total value of $S_{z}$.

For the DMC algorithm we have used the short-time Green function approximation ${ }^{30,31}$ with an $O\left(\tau^{3}\right)$ form. ${ }^{32}$ For those systems involving nodal surfaces, i.e. for fermions, the fixed node approximation has been employed. $\underline{30,33,34}$ The energy accuracy depends on the quality of the nodal surfaces of the trial function, which arise from the Slater determinants. FeynmanCohen back-flow correlations ${ }^{35}$ have been incorporated into the scheme by substituting

$$
\mathbf{r}_{i} \rightarrow \tilde{\mathbf{r}}_{i}=\mathbf{r}_{i}+\sum_{i \neq j} \eta\left(r_{i j}\right)\left(\mathbf{r}_{i}-\mathbf{r}_{j}\right)
$$

in the orbitals of the Slater determinants. $\underline{36}^{-}$For the backflow function $\eta(r)$ we choose the medium-range form used in Ref. 37, namely $\eta(r)=\lambda / r^{3}$, with the same value of $\lambda=5 \AA^{3}$. These type of correlations give rise to nodal surfaces that provide very accurate results for different fermionic systems in Quantum Monte Carlo calculations. $\underline{26}, \underline{38}-\underline{43}$ Pure estimator results discussed later on are free of the trial wave function bias in the density distributions. The accuracy of the wave function only affects the rate of the convergence of the calculation.

As in our previous works on pure and doped mixed helium clusters, $\frac{12,25,26}{2}$ we have fixed the parameters $b_{M N}$ by taking $\epsilon$ as the minimum of the interaction, and $\sigma$ as the distance at which the interaction is zero. Moreover, we have slightly modified the value of the exponent $\nu$ with respect to the McMillan value. All in all, for all cluster sizes we have used the following values: $\nu_{M N}=5.2, b_{44}=2.95 \AA, b_{33}=2.85 \AA, b_{34}=2.90 \AA, b_{4 \mathrm{Mg}}=5.831 \AA$, and $b_{3 \mathrm{Mg}}=5.687 \AA$. Thus, the trial or importance sampling wave function contains only five free parameters, namely $\alpha_{44}, \alpha_{33}, \alpha_{34}, \alpha_{4 \mathrm{Mg}}$, and $\alpha_{3 \mathrm{Mg}}$, which have been determined by minimizing the expectation value of the Hamiltonian. It turns out that for isotopically pure boson $\left(N_{3}=0\right)$ or fermion $\left(N_{4}=0\right)$ clusters, these parameters are well represented in terms of the number of atoms in the drop, namely $\alpha_{44}=0.117-0.029 N_{4}^{1 / 3}, \alpha_{4 \mathrm{Mg}}=$ $0.268-0.011 N_{4}^{1 / 3}, \alpha_{33}=0.020+0.006 N_{3}^{1 / 3}$, and $\alpha_{3 \mathrm{Mg}}=0.191+0407 N_{3}^{1 / 3}-0.152 N_{3}^{2 / 3}$.

Our DMC calculation is based on an importance sampling function, and therefore the walkers are generated according to the auxiliary function $f(\mathcal{R}, t)=\Psi_{T}(\mathcal{R}) \Psi(\mathcal{R}, t)$. The natural output corresponds thus to the so called mixed estimator, in which the expectation value of a given operator is straightforwardly calculated with this probability distribution function. If such an operator commutes with the Hamiltonian, the mixed estimator is equal to the exact expectation value of the observable in the asymptotic limit and within the fixed- 
node error. For other observables, as for example radial operators, the mixed estimator is in general biased by the trial function used for importance sampling. For the reasons mentioned in the Introduction, we have chosen to obtain unbiased estimates of radial distances for some ${ }^{4} \mathrm{He}$ droplets. In view of the few unbiased available calculations, ours may contribute to the general discussion of the subject.

Within Quantum Monte Carlo simulations, several schemes have been proposed in the literature to obtain unbiased - also called pure - estimators of operators that do not commute with the Hamiltonian. In this work we compute pure expectation values by using a forward walking method. The idea is to recover the exact value by including the factor $\Psi(\mathcal{R}, t) / \Psi_{T}(\mathcal{R})$ in the expectation value. This quotient can be obtained starting from the asymptotic offspring of the walker $\underline{\underline{44}}$ Therefore a weight proportional to the number of the future descendant of the walker

$$
W(\mathcal{R}) \propto n(\mathcal{R}, t \rightarrow \infty)
$$

needs to be included in the calculation. We have employed the algorithm devised in Ref. 45 to calculate this weight. It makes use of an auxiliary variable associated with each walker that evolves with it, i.e. it is replicated as many times as the walker and propagates the local values. With the proper boundary conditions, the final average provides the pure expectation value. The basis of the method and details on the algorithm can be found in Refs. 45, 46. The pure estimation depends on the size of the block, $\Delta_{L}$, that needs to be large enough to fulfill the forward walking condition Eq. (66). A study of the convergence as a function of $\Delta_{L}$ is required to fix the block length from which the pure estimator provides the same value within the statistical error for a given radial operator.

\section{RESULTS}

\section{A. Structure of $\mathbf{M g} @{ }^{4} \mathbf{H e}_{N_{4}}+{ }^{3} \mathbf{H e}_{N_{3}}$ clusters}

For illustrative purposes, the mixed estimator total helium particle densities of the ${ }^{4} \mathrm{He}_{40}$, ${ }^{3} \mathrm{He}_{20}+{ }^{4} \mathrm{He}_{20}$ and ${ }^{3} \mathrm{He}_{40}$ droplets doped with $\mathrm{Mg}$ are plotted in Fig. 2, These densities have been obtained as follows. $\stackrel{12}{*}$ After a simulation running for a long thermalization time, we

have stored a large number of walkers (typically $10^{6}$ ) and for each of them the origin is taken at the center of mass of the helium atoms. Next, a rotation is carried out so that the 
$\mathrm{Mg}$ atom lies on the z-axis. In this coordinate system, a projection onto the $y=0$ plane is performed in order to compute the density.

It can be qualitatively seen that, while for ${ }^{3} \mathrm{He}_{40} \mathrm{Mg}$ is fully immersed, for ${ }^{4} \mathrm{He}_{40}$ it is not. We will see in Sec IIC how the distinct helium environment around Mg is reflected in the absorption spectrum for these three complexes.

Figures 3 and 4 represent the mixed estimator densities corresponding to the doped droplets ${ }^{3} \mathrm{He}_{8}+{ }^{4} \mathrm{He}_{20}$ and ${ }^{3} \mathrm{He}_{20}+{ }^{4} \mathrm{He}_{20}$, respectively. It is interesting to notice that the ${ }^{3} \mathrm{He}$ component may get in touch with the impurity. This is even so for the $N_{3}=N_{4}=20$ system, the reason being that the balance between the weak He-He and Mg-He interactions favors that the $\mathrm{Mg}$ atom is not fully coated by ${ }^{4} \mathrm{He}$ for such small droplets and the chosen composition. The situation changes when $N_{4}$ increases $\underline{47}, \underline{48}$

The mixed estimators for the root mean square (rms) radius of the helium cluster and the Mg impurity are plotted in Fig. 5 , We have chosen to refer them to the center-of-mass of the He droplet instead of the center-of-mass of the He+Mg complex, so that the data clearly show where the $\mathrm{Mg}$ sits with respect to the He moiety.

In ${ }^{4} \mathrm{He}$ droplets the $\mathrm{Mg}$ rms radius increases with cluster size for small $\left(N_{4} \lesssim 25\right)$ droplets. One may infer from this that, for small $N_{4}$ values, the impurity sits in the outer region of the droplet. At $N_{4} \simeq 25$ the impurity begins to "sink" into the cluster as its rms radius starts to decrease with increasing cluster size. In ${ }^{3} \mathrm{He}$ droplets no such trend is seen for the $\mathrm{Mg}$ rms radius, which contrarily has a tendency to decrease as the cluster size increases, apart from the structure around $N_{3}=14$ for whose origin, likely related to shell effects, we have been unable to find a convincing explanation. These results are consistent with the finding that $\mathrm{Mg}$ is in the bulk of the droplet for any $N_{3}$ value,,$\frac{10}{-}$ as Ancilotto's criterion predicts.

The transition in the Mg location Fig. 5 hints at can be clearly seen in Fig. 6, where the mixed estimator radial probability distribution of $\mathrm{Mg}$ in ${ }^{4} \mathrm{He}$ clusters is shown for several sizes from $N_{4}=8$ to 30 . For small cluster sizes $\left(N_{4}<25\right)$, the $\mathrm{Mg}$ atom is found at the surface of the droplet. The typical distance between the impurity and the center-of-mass of the droplet is about 3-4 $\AA$. At $N_{4}=25 \mathrm{Mg}$ experiences a transition from a surface to a bulk state in which the probability of finding the impurity inside the droplet becomes significant. For cluster sizes between $N_{4}=25$ and 29, the impurity is highly delocalized, and for $N_{4} \geq 30$ the Mg atom resides inside the droplet. The typical distance between the impurity and the center-of-mass of the droplet decreases as the impurity becomes fully solvated. Our results 
thus confirm those of Mella et al. $\stackrel{14}{\underline{1}}$

Finally, we have employed the pure estimator to determine the radial structure for the isotopically pure $N_{4}=8$ and 30 droplets. We have found that the differences between pure and mixed estimators of the radial densities are very small and not worth to be plotted. As an illustration, we have shown in Fig. [7 the mixed and pure estimations for the rms radius as a function of the block size $\Delta_{L}$. The stability of the results is apparent, as well as the quality of our mixed estimator for radial distances. Thus, the conclusions drawn using the mixed estimator are robust and remain unchanged and accordingly, apart from Fig. 7, the results discussed in this work have all been obtained using the mixed estimator.

\section{B. Energetics of $\mathrm{Mg} @{ }^{4} \mathrm{He}_{N_{4}}+{ }^{3} \mathrm{He}_{N_{3}}$ clusters}

The calculated DMC ground state energies $E\left(\mathrm{Mg} @{ }^{4} \mathrm{He}_{N_{4}}+{ }^{3} \mathrm{He}_{N_{3}}\right)$ are given in Tables $\amalg$, III, and IV for several $\left(N_{3}, N_{4}\right)$ combinations. In these Tables we have shown the statistical error in the last figure obtained in the usual way, as the standard deviation of the mean value calculated by using the blocking method. The solvation energy of the dopant in the droplet, defined as

$$
\mu_{\mathrm{Mg}}=E\left(\mathrm{Mg} @{ }^{4} \mathrm{He}_{N_{4}}+{ }^{3} \mathrm{He}_{N_{3}}\right)-E\left({ }^{4} \mathrm{He}_{N_{4}}+{ }^{3} \mathrm{He}_{N_{3}}\right),
$$

is also given in the Tables. No solvation energy is given for $\mathrm{Mg} @{ }^{3} \mathrm{He}_{N_{3}}$ droplets because pure ${ }^{3}$ He droplets are unbound for such small sizes ${ }^{25,43,49}$ and hence the solvation energy is just the ground state energy of the $\mathrm{Mg} @{ }^{3} \mathrm{He}_{N_{3}}$ complex. At first glance, it is surprising that one single $\mathrm{Mg}$ atom is able to bound any number of ${ }^{3} \mathrm{He}$ atoms, since the $\mathrm{Mg}$-He interaction is weaker than the He-He one, see Fig. 1. The smaller zero-point energy of Mg together with the fact that the equilibrium distance is larger for Mg-He than for He-He causes the extra binding. Thus, the complexes Mg@ ${ }^{4} \mathrm{He}_{N_{4}}, \mathrm{Mg} @{ }^{3} \mathrm{He}_{N_{3}}$ and $\mathrm{Mg} @{ }^{4} \mathrm{He}_{N_{4}}+{ }^{3} \mathrm{He}_{N_{3}}$ are bound for any $N_{3}$ and $N_{4}$ values.

A comparison between our DMC results for $\mathrm{Mg} @{ }^{4} \mathrm{He}_{N_{4}}$ clusters and other DMC results from Refs. 14 and 22 is presented in Table V] Mella et al. $\underline{\underline{4}}$ have used a Mg-He interaction

determined at the CCSDT level, and the He-He interaction of Ref. 50. The differences beyond statistical errors found between our results and theirs could be mostly attributed to minor differences in the interaction potentials. The agreement with Elhyiani ${ }^{22}$-who has 
used the same interactions as in this work- is very satisfactory.

The ground state and Mg solvation energies are presented in Figs. 8 and 9 respectively, as a function of the total number of He atoms. For the sizes considered here, it can be seen that the $\mathrm{Mg}$ solvation energy does not much change when ${ }^{3} \mathrm{He}$ atoms are added to the $\mathrm{Mg} @{ }^{4} \mathrm{He}_{N_{4}}$ droplet.

Both ground state and solvation energies are smooth functions of the total number of helium atoms. It is worthwhile noting that for doped ${ }^{3} \mathrm{He}$ droplets, these energies display conspicuous oscillations. We have defined the ${ }^{3} \mathrm{He}$ separation energy as

$$
S_{3^{\mathrm{He}}}=E\left(\mathrm{Mg} @^{3} \mathrm{He}_{N_{3}-1}\right)-E\left(\mathrm{Mg} @^{3} \mathrm{He}_{N_{3}}\right),
$$

and have plotted this quantity as a function of $N_{3}$ in Fig. 10, $S_{3}$ He has a sawtooth structure similar to that of the atomic ionization energy vs. atomic number ${ }^{51}$ The more tightly bound ${ }^{3} \mathrm{He}$ atoms at $N_{3}=8$ and 20 allows one to identify shell closures, and correlate well with the local minima in Figs. 8 and 9. A similar result was obtained for Ca. $\frac{12}{2}$ This is somewhat an unexpected result, as ${ }^{3} \mathrm{He}$ droplets doped with $\mathrm{Mg}$ atoms look closer to axially than to spherically symmetric systems. We recall that for spin saturated systems, the first shell closures of the three-dimensional spherical harmonic oscillator appear for 2, 8, and 20 spin $1 / 2$ fermions.

\section{Absorption spectrum of $\mathrm{Mg}$ in ${ }^{4} \mathrm{He}_{N_{4}}+{ }^{3} \mathrm{He}_{N_{3}}$ clusters}

It is well-known that the shift and width of the electronic transitions of impurities in helium droplets are very sensitive to their environment and for this reason this technique is often employed to study their structure. $\underline{\underline{5}} \underline{\underline{7}}$ We have calculated the dipole absorption spectrum of $\mathrm{Mg}$ as described in Ref. 12. The line shape of the electronic transition is determined as

$$
I(\omega) \propto \int \mathrm{d} \mathcal{R}\left|\Psi_{g s}(\mathcal{R})\right|^{2} \delta\left(\omega+V_{g s}(\mathcal{R})-V_{e x}(\mathcal{R})\right)
$$

where $\{\mathcal{R}\}$ refers to the positions of the atoms, and $V_{g s}$ and $V_{e x}$ are, respectively, the ground and excited states potential energy surfaces. We recall that we have used the Mg-He $X^{1} \Sigma$ interaction of Ref. 24 for the ground state and the ${ }^{1} \Pi$ and ${ }^{1} \Sigma$ potentials of Ref. 14 for the excited states. To compute $I(\omega)$ for a given value of $\omega$, one has to diagonalize a $3 \times 3$ matrix to determine the three components of the absorption line, each one arising from a different 
potential energy surface, i.e., eigenvalue of the excited energy matrix. We refer the reader to Refs. $52-56$ and references therein for the details.

The DMC calculation provides us with a set of walkers $\left\{\mathcal{R}_{j}\right\}$ representing the instantaneous position of each atom in the cluster. These walkers have been used for determining the one-body densities presented before and can be also employed to obtain the absorption spectrum replacing $\int \mathrm{d} \mathcal{R}\left|\Psi_{g s}(\mathcal{R})\right|^{2}$ by a sum over $\left\{\mathcal{R}_{j}\right\}$ in the above equation, so that a mixed estimator of $I(\omega)$ has been obtained. This is the same approximation employed for the calculation of the radial densities here studied finding no significant differences with their pure estimator values.

As an illustrative example, the absorption spectrum of $\mathrm{Mg}$ is plotted in Fig. 11 for three selected $\left(N_{3}, N_{4}\right)$ combinations with $N_{3}+N_{4}=40$. The spectra are referred to that of $\mathrm{Mg}$ in the gas phase. As for other impurities, the long tail at high frequencies arises from the very repulsive contribution of the ${ }^{1} \Sigma$ pair potential. The largest atomic shift corresponds to the ${ }^{4} \mathrm{He}$ droplet and the smallest one to the ${ }^{3} \mathrm{He}$ droplet, the one corresponding to the mixed droplet lies in between.

The relative value of the shifts is easy to understand from the appearance of the helium densities shown in Fig. 2, Roughly speaking, the larger the density around the impurity, the larger the shift. For $N_{3}+N_{4}=40, \mathrm{Mg}$ is fairly coated by helium and the value of the saturation density plays a significant role in the actual value of the shift. Since the saturation density is larger for ${ }^{4} \mathrm{He}$ than for ${ }^{3} \mathrm{He}$ this explains the relative position of the three absorption lines.

\section{SUMMARY}

Using a DMC approach, we have found that a $\mathrm{Mg}$ atom in a ${ }^{4} \mathrm{He}$ droplet experiences a transition from a surface to a bulk location for $N_{4} \geq 26$. For larger ${ }^{4}$ He droplets, the impurity resides in the bulk of the droplet. This conclusion has been drawn not only using the mixed estimator inherent to the importance sampling approach, but also using a pure estimator approach free from this bias. This finding agrees with the result obtained by Mella and coworkers, $\stackrel{14}{\underline{1}}$ with which only minor quantitative differences are found.

Due to the light mass of this alkaline earth atom and its weak interaction with helium, Mg impurities are found to be very delocalized inside droplets containing several thousand 
${ }^{4} \mathrm{He}$ atoms,,$\frac{13}{}$ as those of interest in recent experiments. ${ }^{18,19}$ In the case of ${ }^{3} \mathrm{He}$, we have found that $\mathrm{Mg}$ is always in the bulk of the ${ }^{3} \mathrm{He}$ droplet, as determined by previous DFT calculations. 10 To the best of our knowledge, the present calculations are the only DMC ones available for this system and for isotopically mixed helium droplets doped with $\mathrm{Mg}$ as well.

For mixed droplets, we have found the well-known scenario $\underline{47}, \underline{48}$ that ${ }^{3} \mathrm{He}$ and ${ }^{4} \mathrm{He}$ atoms are distributed around the impurity into a onion-like shell structure, with ${ }^{4} \mathrm{He}$ atoms coating the Mg impurity. This happens once the number of ${ }^{4} \mathrm{He}$ atoms is large enough to fully cover the dopant. For small $N_{4}$ values, it may appear that some ${ }^{3} \mathrm{He}$ is in contact with the $\mathrm{Mg}$ atom, which is again a consequence of the surface location of $\mathrm{Mg}$ in small ${ }^{4} \mathrm{He}$ clusters. We want to point out, however, that distinguishing surface from volume regions in such small systems is largely arbitrary.

The DMC walkers have been employed to calculate the dipole absorption line taking as a case of study three selected clusters with $N_{3}+N_{4}=40$. We have found that the atomic shift is different enough for the studied configurations. This result only pertains to small mixed droplets. Indeed, when the number of ${ }^{4} \mathrm{He}$ atoms in the droplet is large enough, the shift will be insensitive to the actual composition of the droplet, as the ${ }^{3} \mathrm{He}$ component will be distributed into a shell distant from the impurity, whose absorption line will quickly tend to that of $\mathrm{Mg}$ in liquid ${ }^{4} \mathrm{He} \cdot{ }^{16,17}$

\section{Acknowledgments}

This work has been supported by Grants FIS2007-60133, FIS2008-00421/FIS and FIS2009-07390 from DGI, Spain (FEDER), 2009SGR1289 from Generalitat de Catalunya, and by the Junta de Andalucía. DM has been supported by the ME (Spain) FPU program, Grant No. AP2008-04343.

1 O. Bünermann, G. Droppelmann, A. Hernando, R. Mayol, and F. Stienkemeier, J. Phys. Chem. A 111, 12684 (2007).

2 D. Mateo, A. Hernando, M. Barranco, R. Mayol, and M. Pi, Phys. Rev. B 83, 174505 (2011).

3 J. P. Toennies and A. F. Vilesov, Angew. Chem. Int. Ed. 43, 2622 (2004). 
4 M. Barranco, R. Guardiola, E. S. Hernández, R. Mayol, J. Navarro, and M. Pi, J. Low Temp. Phys. 142, 1 (2006).

5 F. Stienkemeier and K. K. Lehman, J. Phys. B: At. Mol. Opt. Phys. 39, R127 (2006).

6 M. Y. Choi, G. E. Douberly, T. M. Falconer, W. K. Lewis, C. M. Lindsay, J. M. Merrit, P. L. Stiles, and R. E. Miller, Int. Rev. Phys. Chem. 25, 15 (2006).

7 J. Tiggesbäumker and F. Stienkemeier, Phys. Chem. Chem. Phys. 9, 4748 (2007).

8 K. Szalewicz, Int. Rev. Phys. Chem. 27, 273 (2008).

9 F. Ancilotto, P. B. Lerner, and M. W. Cole, J. Low Temp. Phys. 101, 1123 (1995).

10 A. Hernando, R. Mayol, M. Pi, M. Barranco, F. Ancilotto, O. Bünermann, and F. Stienkemeier, J. Phys. Chem. A 111, 7303 (2007).

11 O. Bünermann, M. Dvorak, F. Stienkemeier, A. Hernando, R. Mayol, M. Pi, M. Barranco, and F. Ancilotto, Phys. Rev. B 79, 214511 (2009).

12 R. Guardiola, J. Navarro, D. Mateo, and M. Barranco, J. Chem. Phys. 131, 174110 (2009).

13 A. Hernando, M. Barranco, R. Mayol, M. Pi, and F. Ancilotto, Phys. Rev. B 78, 184515 (2008).

14 M. Mella, G. Calderoni, and F. Cargnoni, J. Chem. Phys. 123, 054328 (2005).

15 J. Reho, U. Merker, M. R. Radcliff, K. K. Lehmann, and G. Scoles, J. Chem. Phys. 112, 8409 (2000).

16 Y. Moriwaki and N. Morita, Eur. Phys. J. D 5, 53 (1999).

17 Y. Moriwaki, K. Inui, K. Kobayashi, F. Matsushima, and N. Morita, J. Mol. Struc. 786, 112 (2006).

18 A. Przystawik, S. Göde, T. Döppner, J. Tiggesbäumker, and K-H. Meiwes-Broer, Phys. Rev. A 78, 021202(R) (2008).

19 Y. Ren and V. V. Kresin, Phys. Rev. A 76, 043204 (2007).

20 M. Elhiyani and M. Lewerenz, contribution to the XXII International Symposium on Molecular Beams, University of Freiburg (2007).

21 M. Elhiyani and M. Lewerenz, contribution to the 16th. Symposium on Atomic and Surface Physics and Related Topics, Innsbruck University Press (2008).

22 M. Elhiyani. PhD thesis, Université Paris-Est, 2009. Unpublished.

23 R. A. Aziz, F. R. McCourt and C. C. K. Wong, Mol. Phys. 61, 1487 (1987).

24 R. J. Hinde, J. Phys. B: At. Mol. Opt. Phys. 36, 3119 (2003).

25 R. Guardiola and J. Navarro, Phys. Rev. Lett. 84, 1144 (2000). 
R. Guardiola and J. Navarro, Phys. Rev. Lett. 89, 193401 (2002).

27 W. L. McMillan, Phys. Rev. 138, A442 (1965).

28 E. Lipparini, Modern Many-Particle Physics, 2nd. edition. World Scientific, Singapore (2008). Section 5.3.

29 G. D. Mahan, Many-Particle Physics, 3rd. edition. Kluwer Academic, New York (2008). Section 11.3.4.

30 P. J. Reynolds, D. M. Ceperley, B. J. Alder, and W. A. Lester Jr., J. Chem. Phys. 77, 5593 $(1982)$.

31 J. B. Anderson, J. Chem. Phys. 73, 3897 (1980).

32 J. Vrbik and S. M. Rothstein, J. Comput. Phys. 63, 130 (1986).

33 J. B. Anderson, J. Chem. Phys. 63, 1499 (1975).

34 J. B. Anderson, J. Chem. Phys. 65, 4121 (1976).

35 R. P. Feynman and M. Cohen, Phys. Rev. 102, 1189 (1956).

36 K. E. Schmidt, M. A. Lee, M. H. Kalos, and G. V. Chester, Phys. Rev. Lett. 47, 807 (1981).

37 V. R. Pandharipande, S. C. Pieper, and R. B. Wiringa, Phys. Rev. B 34, 4571 (1986).

38 J. Casulleras and J. Boronat, Phys. Rev. Lett. 84, 3121 (2000).

39 W.M.C. Foulkes, L. Mitas, R.J. Needs, and G. Rajagopal, Rev. Mod. Phys 73, 33 (2001).

40 L. Brualla, S. Fantoni, A. Sarsa, K.E. Schmidt, and S.A. Vitiello, Phys. Rev. C 67, 065806 (2003).

41 P. López-Ríos, A. Ma, N.D. Drummond, M.D. Towler, and R.J. Needs, Phys. Rev. E 74, 066701 (2006).

42 W.D. Parker, J.W. Wilkins, and R.G. Hennig, Phys. Status. Solidi B 248, 267 (2011).

43 E. Sola, J. Casulleras, and J. Boronat, Phys. Rev. B 73, 092515 (2006).

44 K.S. Liu, M.H. Kalos, and G.V. Chester, Phys. Rev. A 10, 303 (1974).

45 J. Boronat and J. Casulleras, Phys. Rev. B 52, 3654 (1995).

46 A. Sarsa, J. Boronat and J. Casulleras, J. Chem. Phys. 116, 5956 (2002).

47 S. Grebenev, J. P. Toennies, and A. F. Vilesov, Science 279, 2083 (1998).

48 M. Pi, R. Mayol, and M. Barranco, Phys. Rev. Lett. 82, 3093 (1999).

49 M. Barranco, J. Navarro and A. Poves, Phys. Rev. Lett. 78, 4729 (1997).

50 K. Tang, J.P. Toennies and C.L. Yiu, Phys. Rev. Lett. 74, 1546 (1995).

51 R. Eisberg and R. Resnick, Quantum Physics of Atoms, Molecules, Solids, Nuclei and Particles, 
J. Whiley and Sons, New York 2nd. edition (1985).

52 E. Cheng and K. B. Whaley, J. Chem. Phys. 104, 3155 (1996)

53 A. Nakayama and K. Yamashita, J. Chem. Phys. 114, 780 (2001)

54 M. Mella, M. C. Colombo, and G. Morosi, J. Chem. Phys. 117, 9695 (2002)

55 A. Hernando, R. Mayol, M. Pi, and M. Krośnicki, Phys. Rev. B 77, 024513 (2008).

56 A. Hernando, M. Barranco, R. Mayol, M. Pi, F. Ancilotto, O. Bünermann, and F. Stienkemeier, J. Low Temp. Phys. 158, 105 (2010). 
TABLE I: Parameters of the fit to the calculated Mg-He pair potentials. The values are such that Eq. (2) yields the pair potential in $\mathrm{K}$ when the distance $r$ is expressed in $\AA$.

\begin{tabular}{cccc}
\hline & $X^{1} \Sigma($ Ref. 24) & ${ }^{1} \Pi$ (Ref. 14) & ${ }^{1} \Sigma($ Ref. 14) \\
\hline$A$ & $1.16902 \times 10^{7}$ & $1.1308 \times 10^{5}$ & $8.6195 \times 10^{3}$ \\
$\alpha$ & 3.0188 & 1.6555 & 0.00291 \\
$\beta$ & - & 0.17578 & 0.14011 \\
$D$ & 10.002 & 9.3539 & 21.075 \\
$C_{6}$ & $1.9454 \times 10^{4}$ & $2.0241 \times 10^{5}$ & - \\
$C_{8}$ & $1.04717 \times 10^{7}$ & - & - \\
$C_{10}$ & - & $3.5046 \times 10^{8}$ & $2.3161 \times 10^{10}$ \\
\hline
\end{tabular}


TABLE II: Ground state energies of $\mathrm{Mg} @{ }^{4} \mathrm{He}_{N_{4}}$ and $\mathrm{Mg}$ solvation energy (in K).

\begin{tabular}{ccc}
\hline$N_{4}$ Ground state energy & $\mu_{\mathrm{Mg}}$ \\
\hline 8 & $-15.91(1)$ & $-10.80(2)$ \\
20 & $-52.46(2)$ & $-18.52(7)$ \\
21 & $-55.99(2)$ & $-19.23(5)$ \\
22 & $-59.35(2)$ & $-19.62(6)$ \\
23 & $-62.84(2)$ & $-19.85(7)$ \\
24 & $-66.44(2)$ & $-20.33(9)$ \\
25 & $-70.07(2)$ & $-20.57(8)$ \\
26 & $-73.75(2)$ & $-20.99(9)$ \\
27 & $-77.46(2)$ & $-21.46(9)$ \\
28 & $-81.13(2)$ & $-21.89(9)$ \\
29 & $-84.87(2)$ & $-22.17(7)$ \\
30 & $-88.74(2)$ & $-22.63(10)$ \\
40 & $-128.96(3)$ & $-25.83(12)$ \\
\hline
\end{tabular}


TABLE III: Ground state energies of $\mathrm{Mg} @{ }^{4} \mathrm{He}_{N_{4}}+{ }^{3} \mathrm{He}_{N_{3}}$ and $\mathrm{Mg}$ solvation energy (in $\mathrm{K}$ ).

\begin{tabular}{cccc}
\hline$N_{4}$ & $N_{3}$ & Ground state energy & $\mu_{\mathrm{Mg}}$ \\
\hline 2 & 2 & $-5.312(6)$ & $-5.201(6)$ \\
8 & 8 & $-28.77(2)$ & $-16.89(2)$ \\
20 & 8 & $-69.73(4)$ & $-23.03(4)$ \\
8 & 20 & $-43.13(2)$ & $-22.62(3)$ \\
20 & 20 & $-88.51(5)$ & $-26.25(6)$ \\
\hline
\end{tabular}


TABLE IV: Ground state energies of $\mathrm{Mg} @{ }^{3} \mathrm{He}_{N_{3}}$ (in K).

\begin{tabular}{|c|c|c|c|}
\hline \multicolumn{2}{|c|}{$N_{3}$ Ground state energy } & \multicolumn{2}{|c|}{$N_{3}$ Ground state energy } \\
\hline 2 & $-1.887(2)$ & 13 & $-9.31(1)$ \\
\hline 3 & $-2.544(3)$ & 14 & $-9.67(1)$ \\
\hline 4 & $-3.312(3)$ & 15 & $-10.64(1)$ \\
\hline 5 & $-4.206(4)$ & 16 & $-11.61(1)$ \\
\hline 6 & $-5.124(4)$ & 17 & $-12.76(1)$ \\
\hline 7 & $-6.141(5)$ & 18 & $-13.89(1)$ \\
\hline 8 & $-7.266(5)$ & 19 & $-15.19(1)$ \\
\hline 9 & $-7.666(6)$ & 20 & $-16.45(1)$ \\
\hline 10 & $-8.055(7)$ & 21 & $-16.820(1)$ \\
\hline 11 & $-8.508(7)$ & 40 & $-33.57(3)$ \\
\hline 12 & $-8.885(8)$ & & \\
\hline
\end{tabular}


TABLE V: Comparison of several DMC results for $\mathrm{Mg} @{ }^{4} \mathrm{He}_{N_{4}}$ clusters.

\begin{tabular}{r|rrr|lll}
\hline & \multicolumn{3}{|c|}{ Cluster energy (K) } & \multicolumn{3}{c}{ Mg solvation energy (K) } \\
\hline$N_{4}$ & This work & Ref. 14 & Ref. 22 & This work & Ref. 14 & Ref. 22 \\
\hline 8 & $-15.91(1)$ & & $-15.91(1)$ & $-10.80(2)$ & $-10.76(1)$ \\
20 & $-52.46(2)$ & $-51.64(1)$ & & $-18.52(7)$ & $-18.49(1)$ \\
25 & $-70.07(2)$ & $-69.04(1)$ & $-70.15(6)$ & $-20.57(8)$ & $-25.03(4)$ & $-20.93(9)$ \\
30 & $-88.74(2)$ & $-87.41(1)$ & & $-22.63(10)$ & $-26.89(4)$ \\
40 & $-128.96(3)$ & $-126.51(3)$ & $-25.83(12)$ & $-25.39(4)$ \\
\hline
\end{tabular}



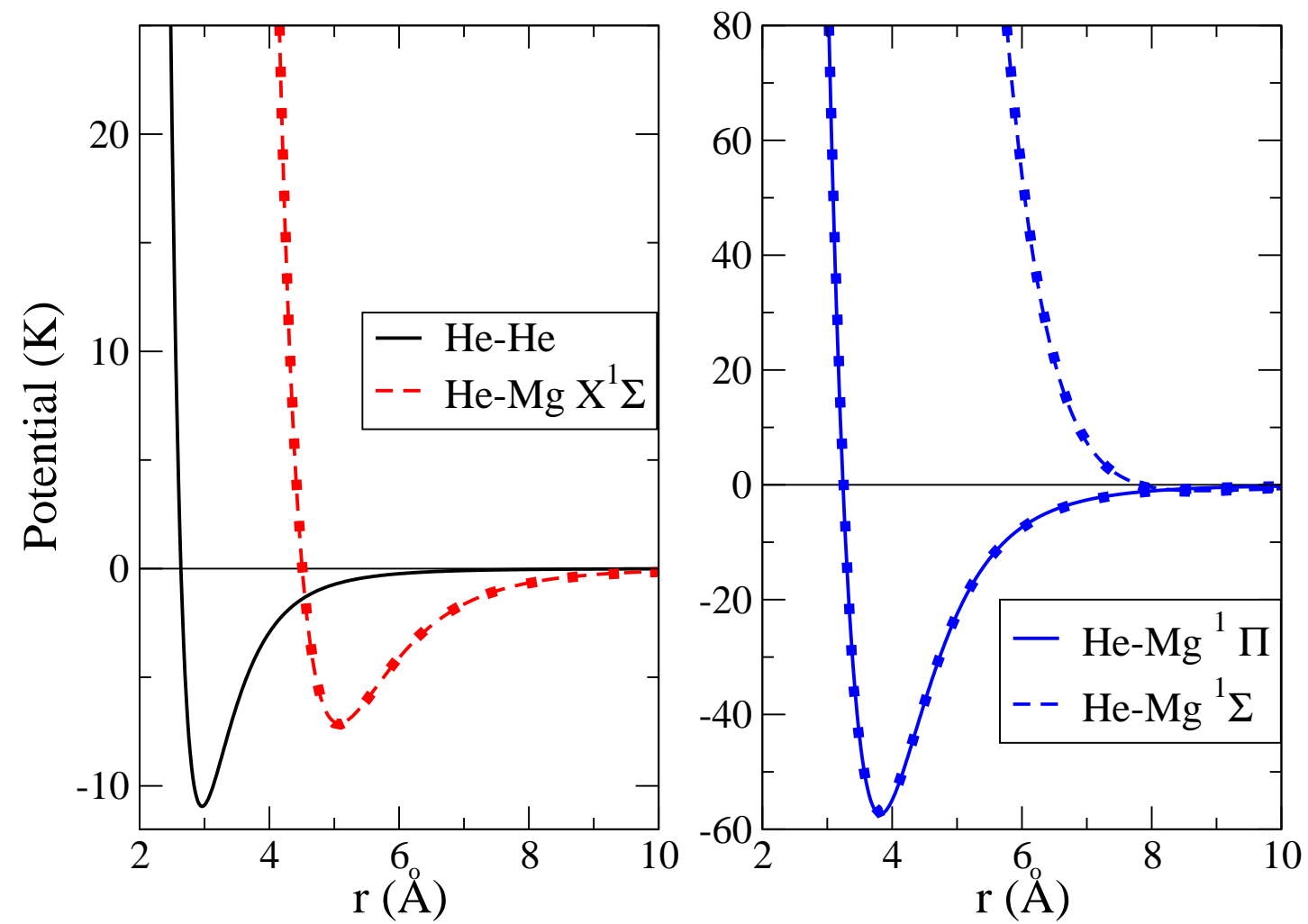

FIG. 1: (Color online) Pair potentials used in this work. The symbols correspond to the results of Hinde $\underline{24}$ for the ground state (left panel) and Mella et al $\stackrel{14}{\underline{14}}$ for the excited states (right panel). The lines correspond to the parameterizations given in Table \. The Aziz He-He potential ${ }^{23}$ has also been plotted in the left panel (solid line). 

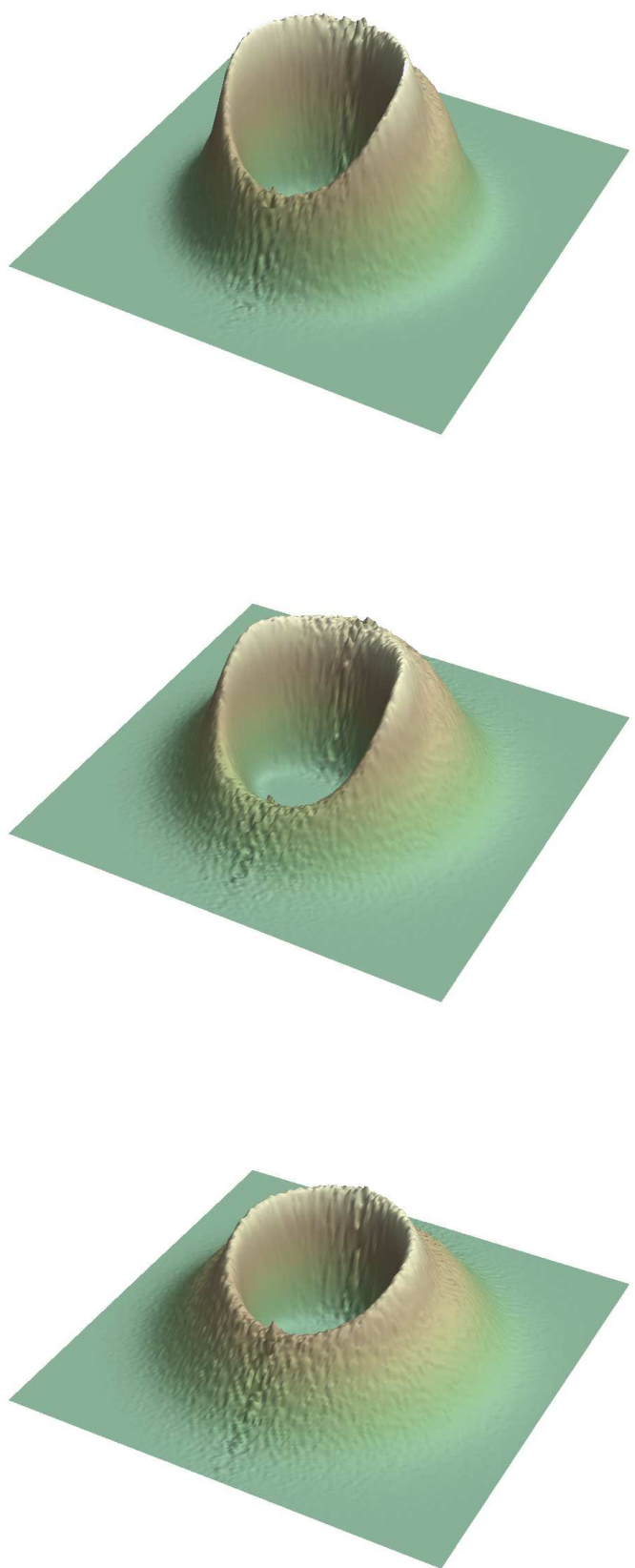

FIG. 2: (Color online) Three-dimensional views of the total He atom density for three droplets doped with Mg. From top to bottom: ${ }^{4} \mathrm{He}_{40},{ }^{3} \mathrm{He}_{20}+{ }^{4} \mathrm{He}_{20}$, and ${ }^{3} \mathrm{He}_{40}$. 

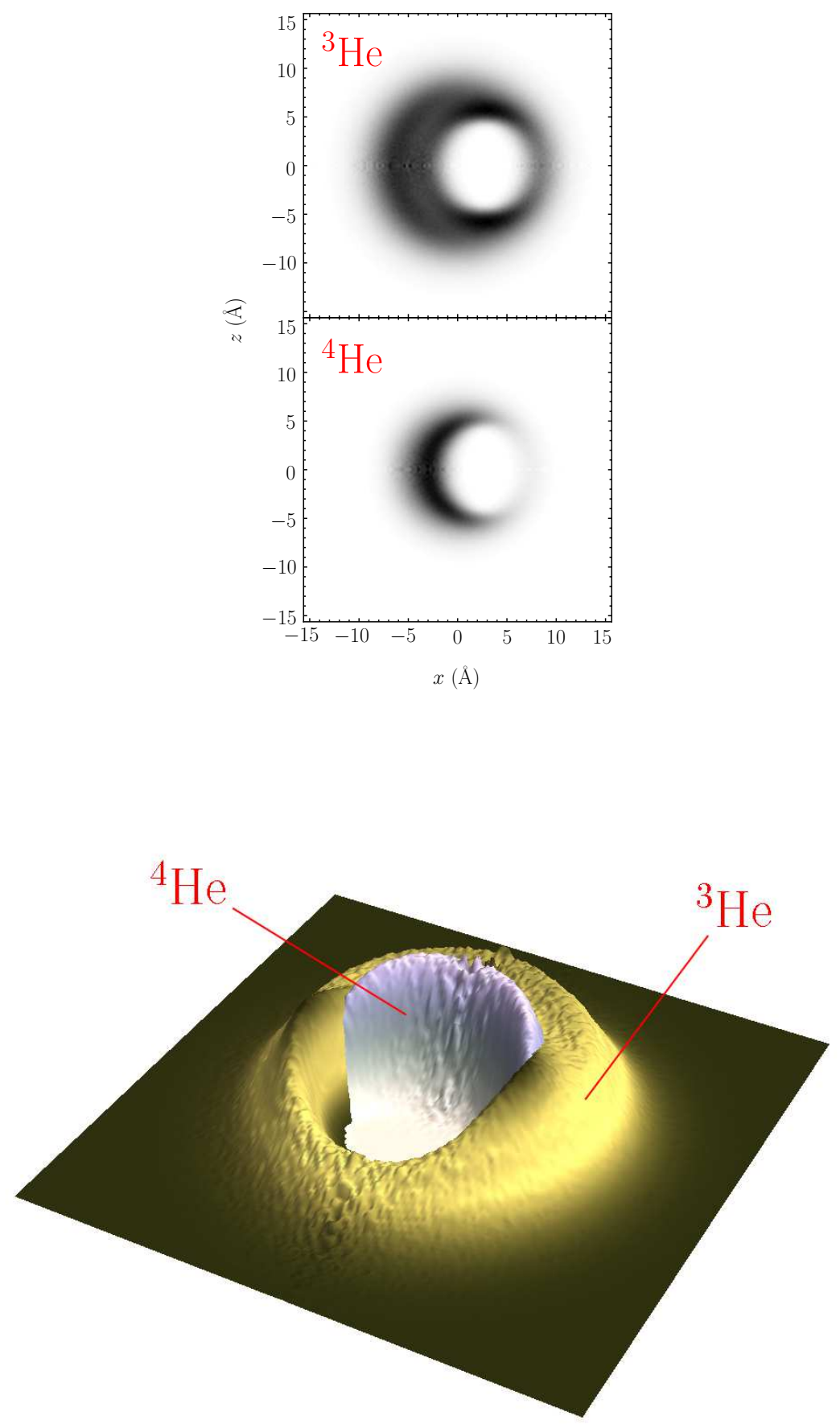

FIG. 3: (Color online) Atomic density of the mixed drop with $N_{4}=8, N_{3}=20$. The contour plots of ${ }^{3} \mathrm{He}$ and ${ }^{4} \mathrm{He}$ densities are separately displayed at the top of the figure. The darker the region, the higher the density. The three-dimensional plot of the same densities is displayed at the bottom of the figure, with the same plot box as in the top contours. 

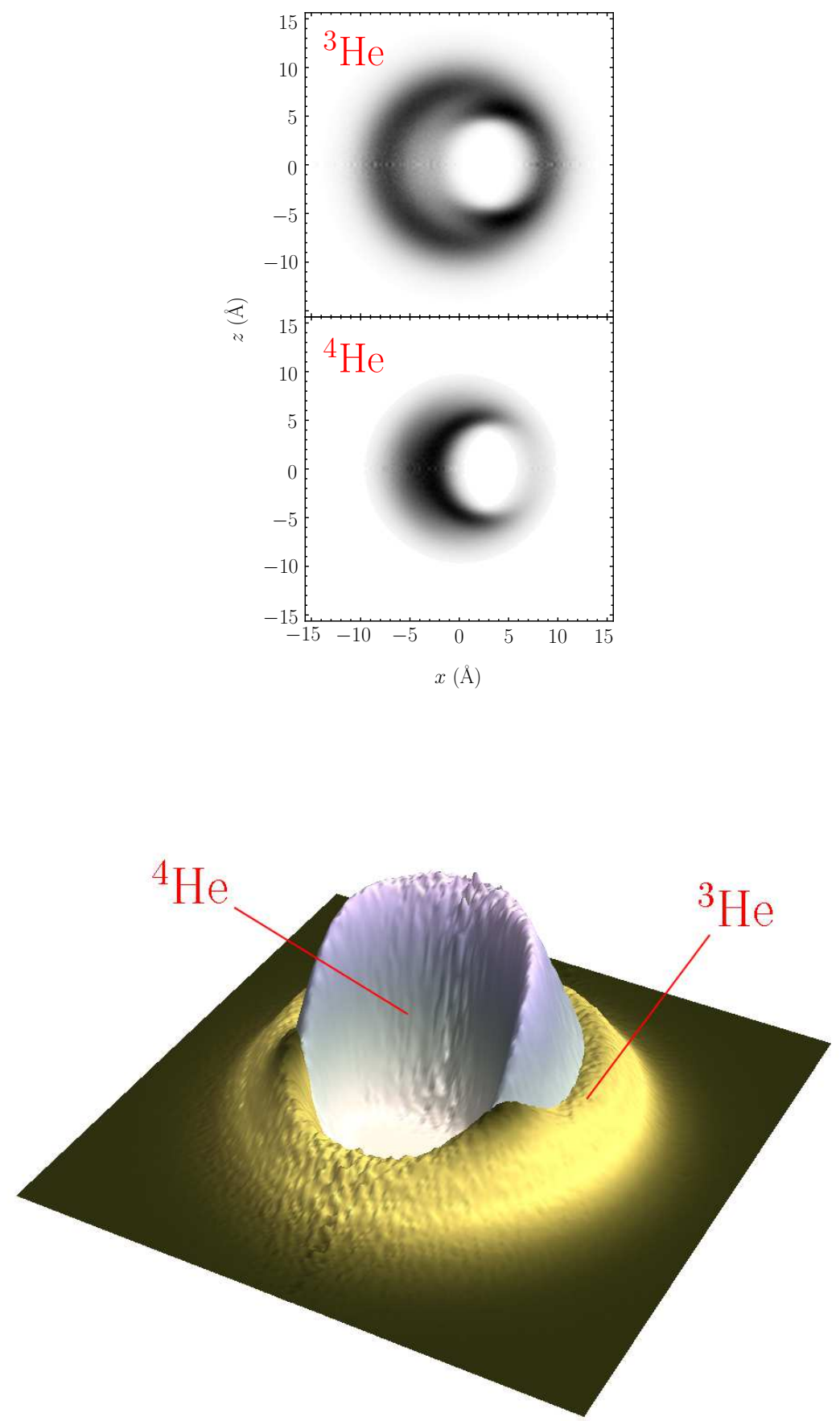

FIG. 4: (Color online) Same as Fig. 3 for $N_{4}=20, N_{3}=20$. 


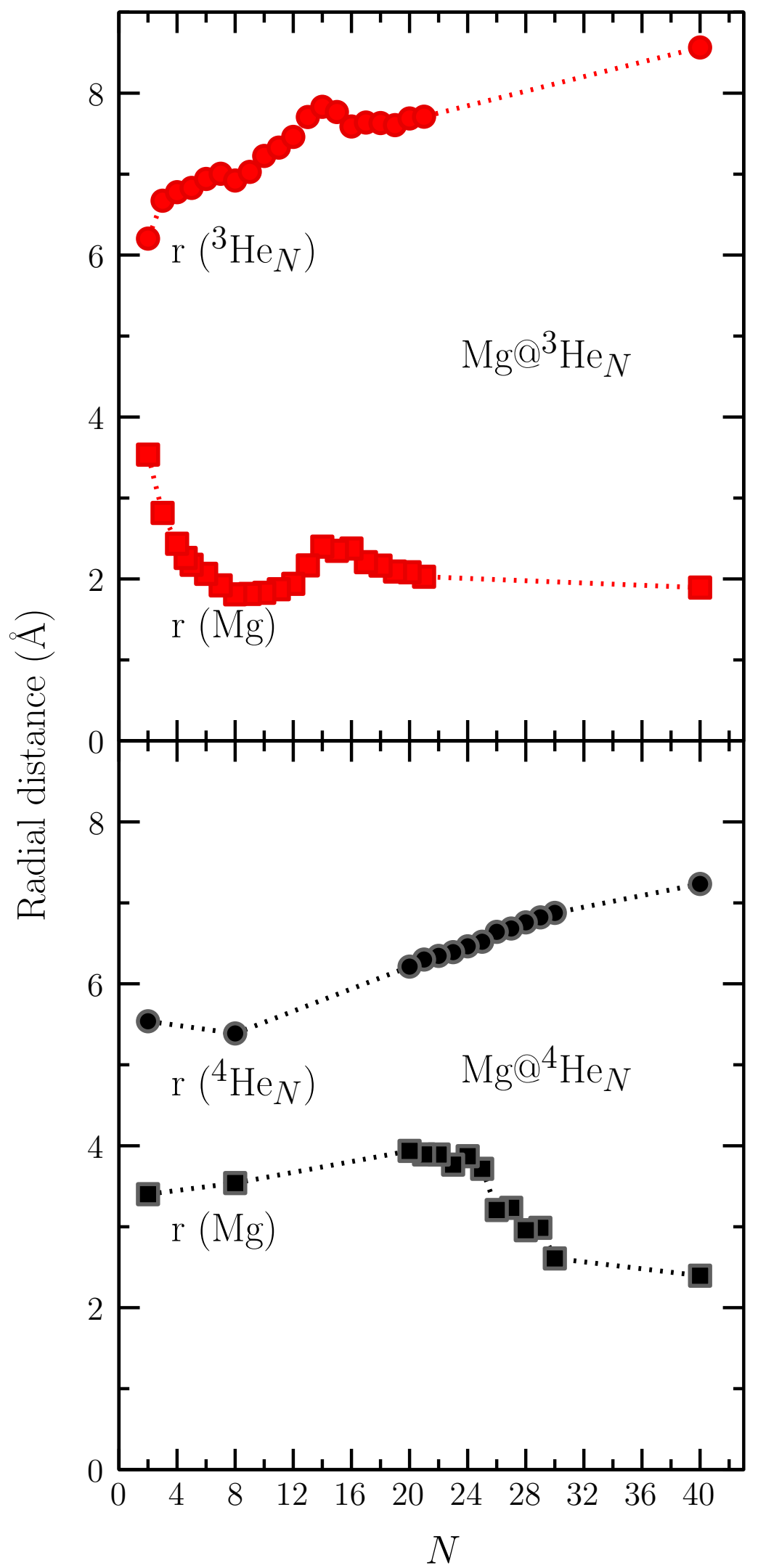

FIG. 5: Root mean square radii of the He clusters and the Mg impurity, measured with respect to the center-of-mass of the He cluster in both cases. The dotted lines are to guide the eye. 


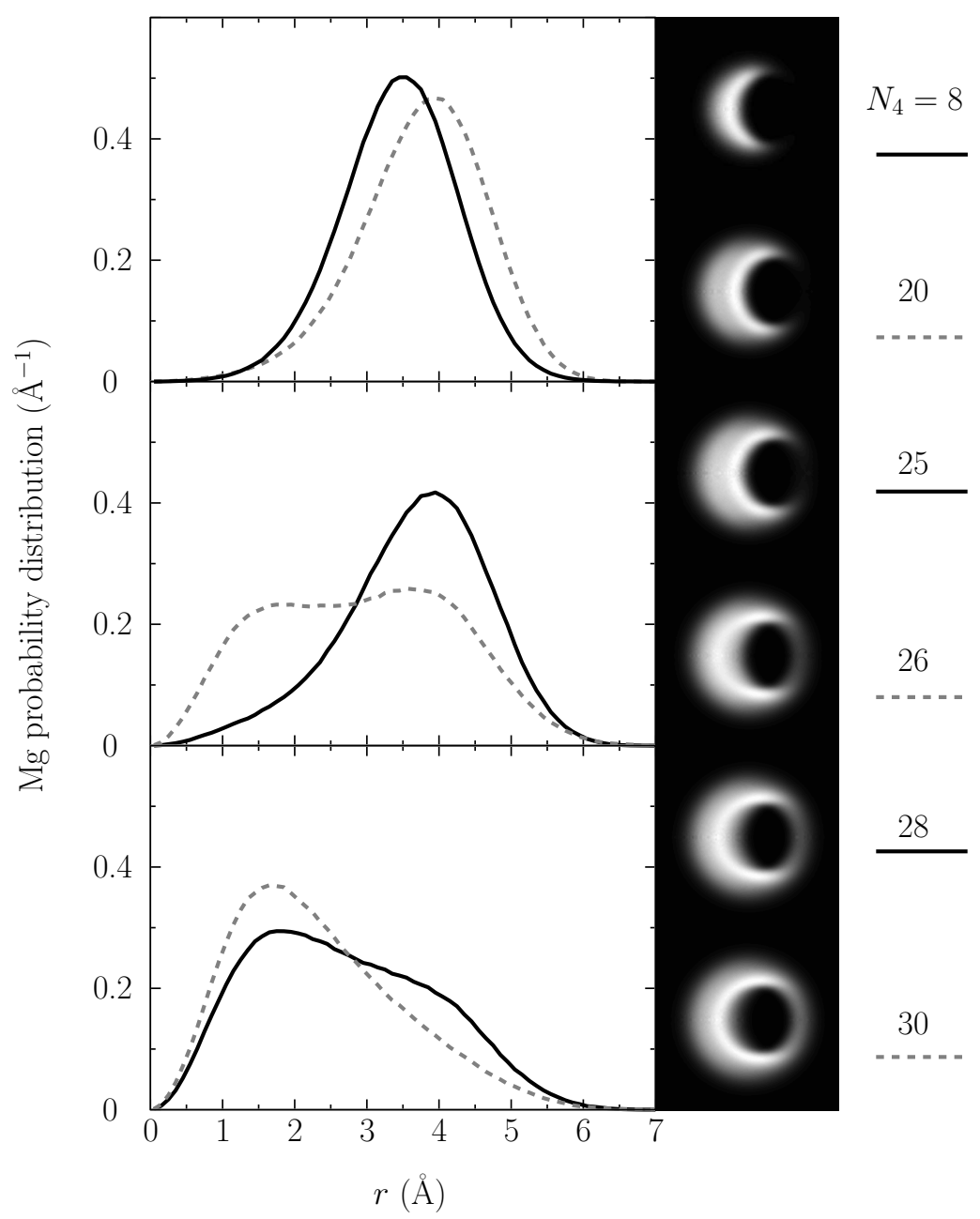

FIG. 6: Grey scale two-dimensional plot of the particle density (right panel) for different $\mathrm{Mg} @{ }^{4} \mathrm{He}_{N_{4}}$ clusters; the lighter the region, the higher the helium density. The He densities are displayed in boxes of $30 \AA \times 30 \AA$. The left panels represent the probability distribution $P(r)$ of finding the $\mathrm{Mg}$ atom at a distance $r$ from the He center-of-mass $\left[\int_{0}^{\infty} d r P(r)=1\right]$. 


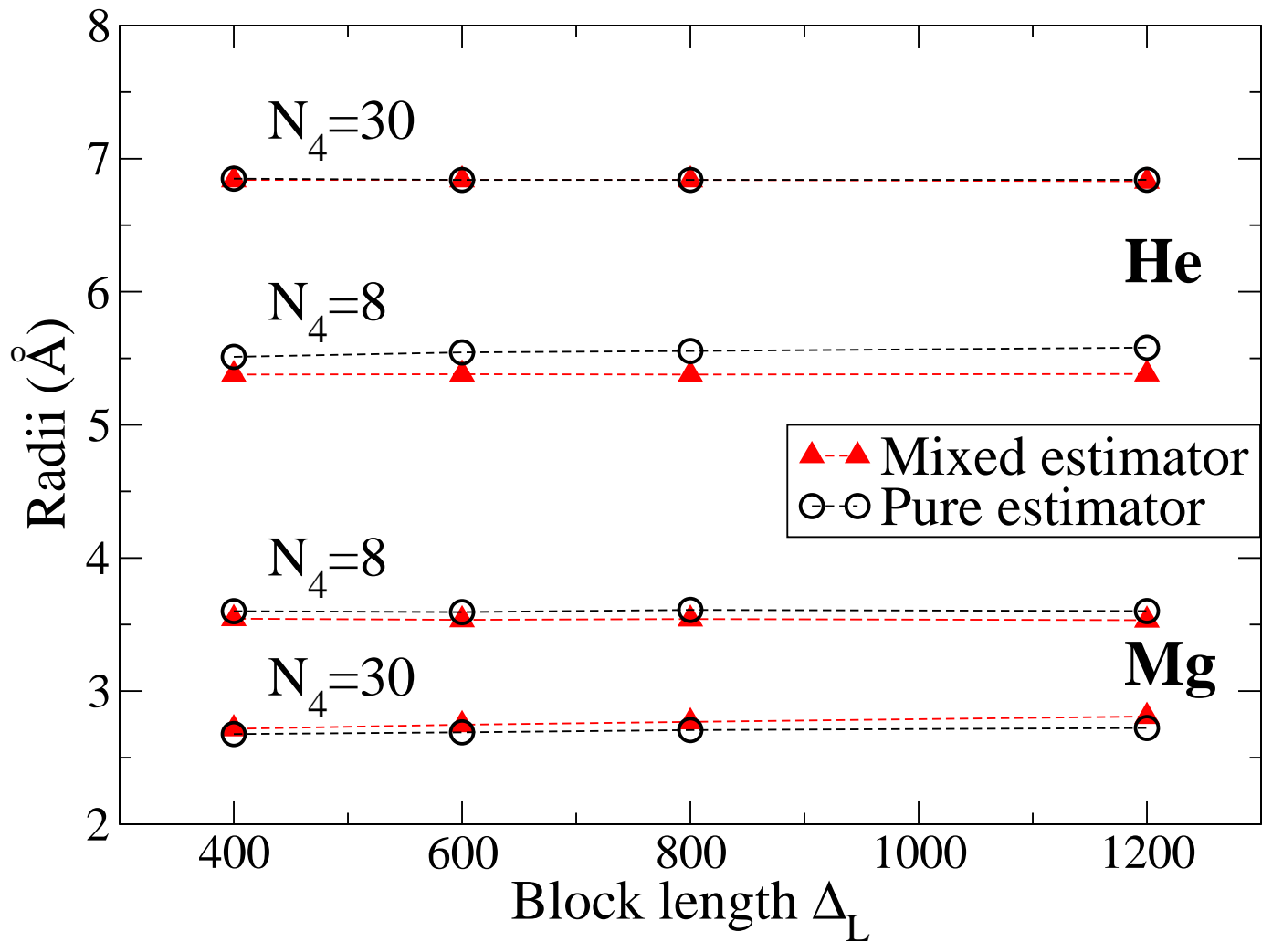

FIG. 7: (Color online) Evolution of the rms radii with the length of blocks of the pure estimator. The dashed lines are to guide the eye. 


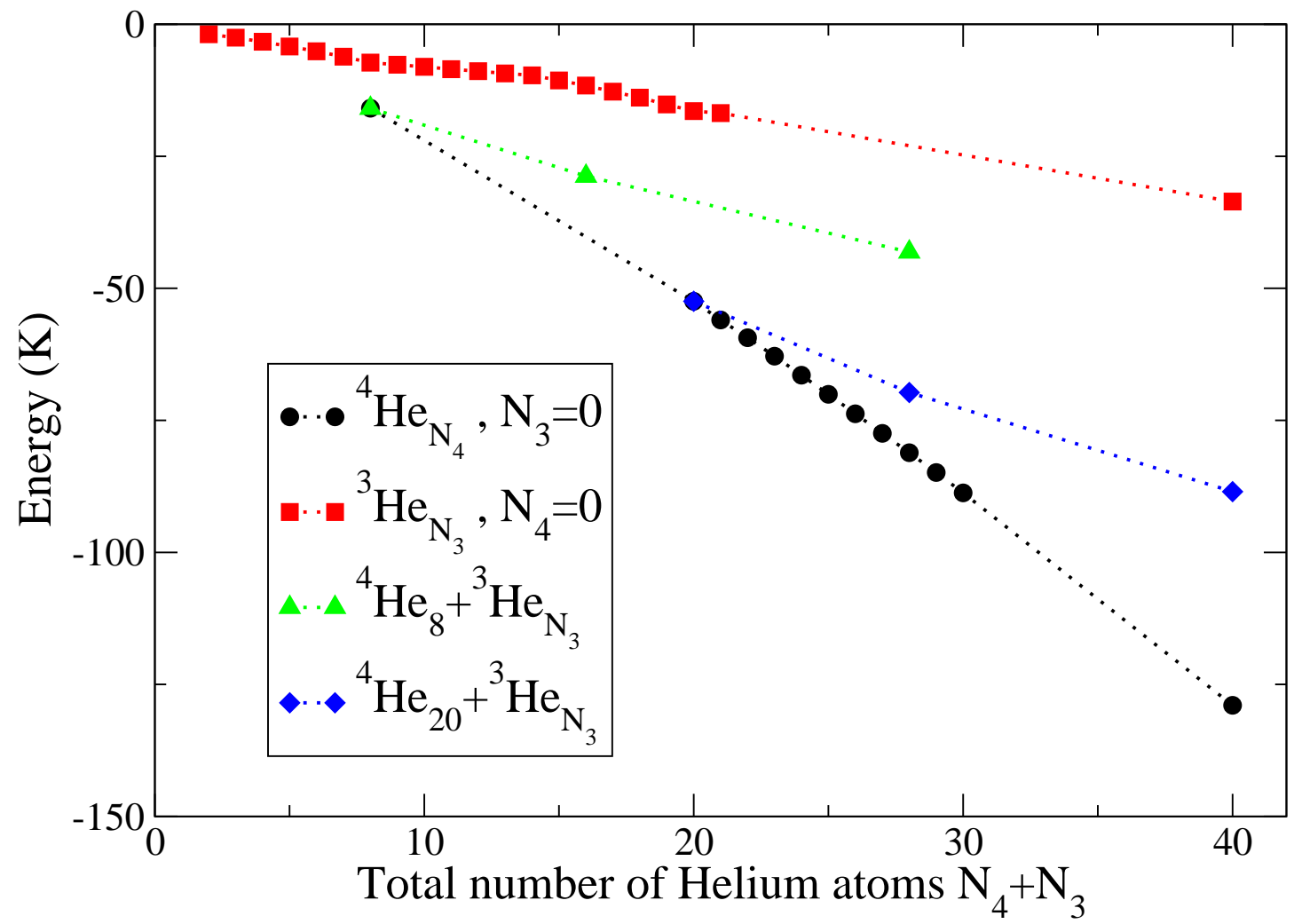

FIG. 8: (Color online) Ground state total energies (in K) of $\mathrm{Mg} @{ }^{4} \mathrm{He}_{N_{4}}+{ }^{3} \mathrm{He}_{N_{3}}$ droplets. The dotted lines are to guide the eye. 


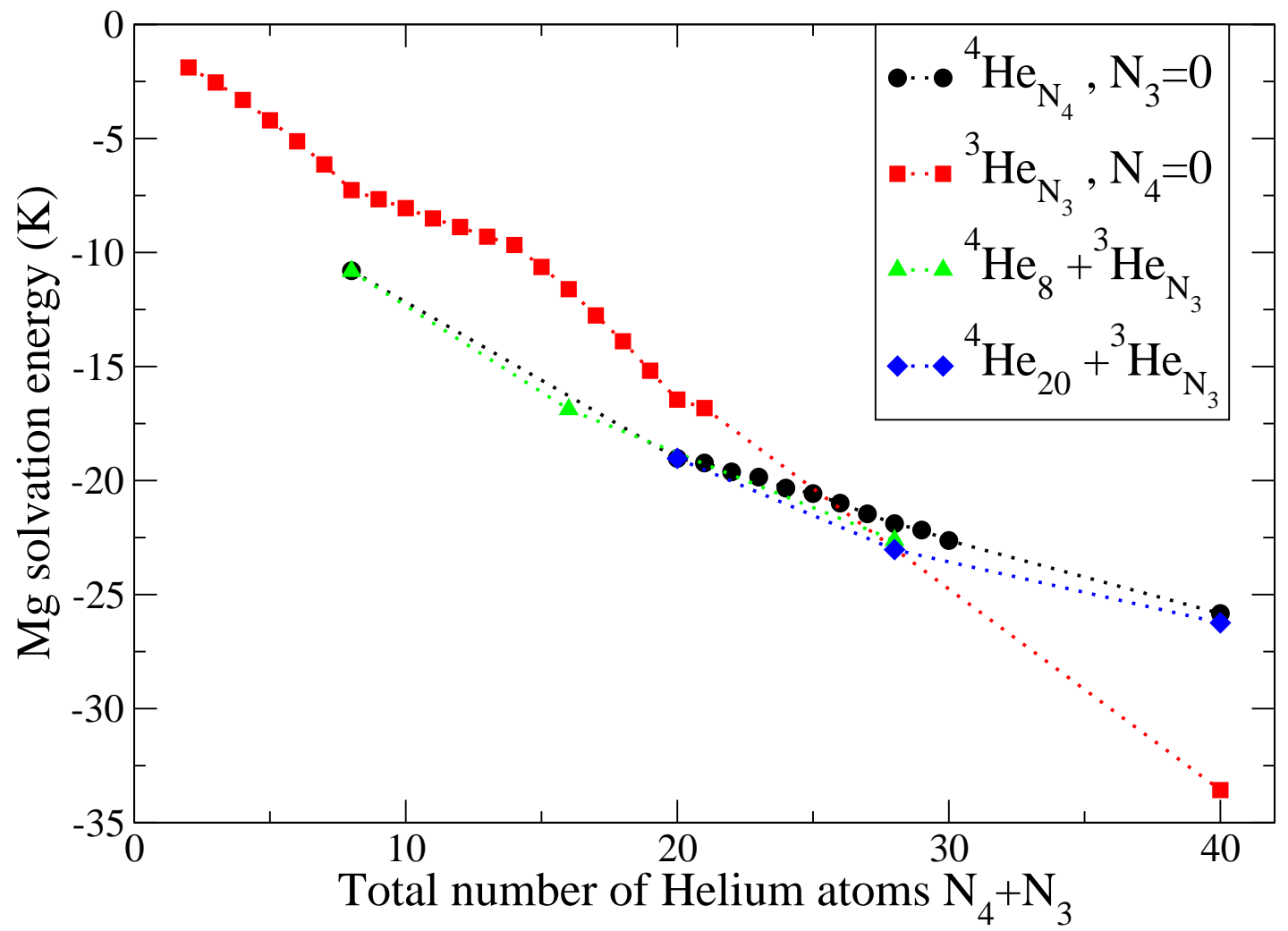

FIG. 9: (Color online) Solvation energy of $\mathrm{Mg}$ (in K) in $\mathrm{Mg} @{ }^{4} \mathrm{He}_{N_{4}}+{ }^{3} \mathrm{He}_{N_{3}}$ droplets. The dotted lines are to guide the eye. 


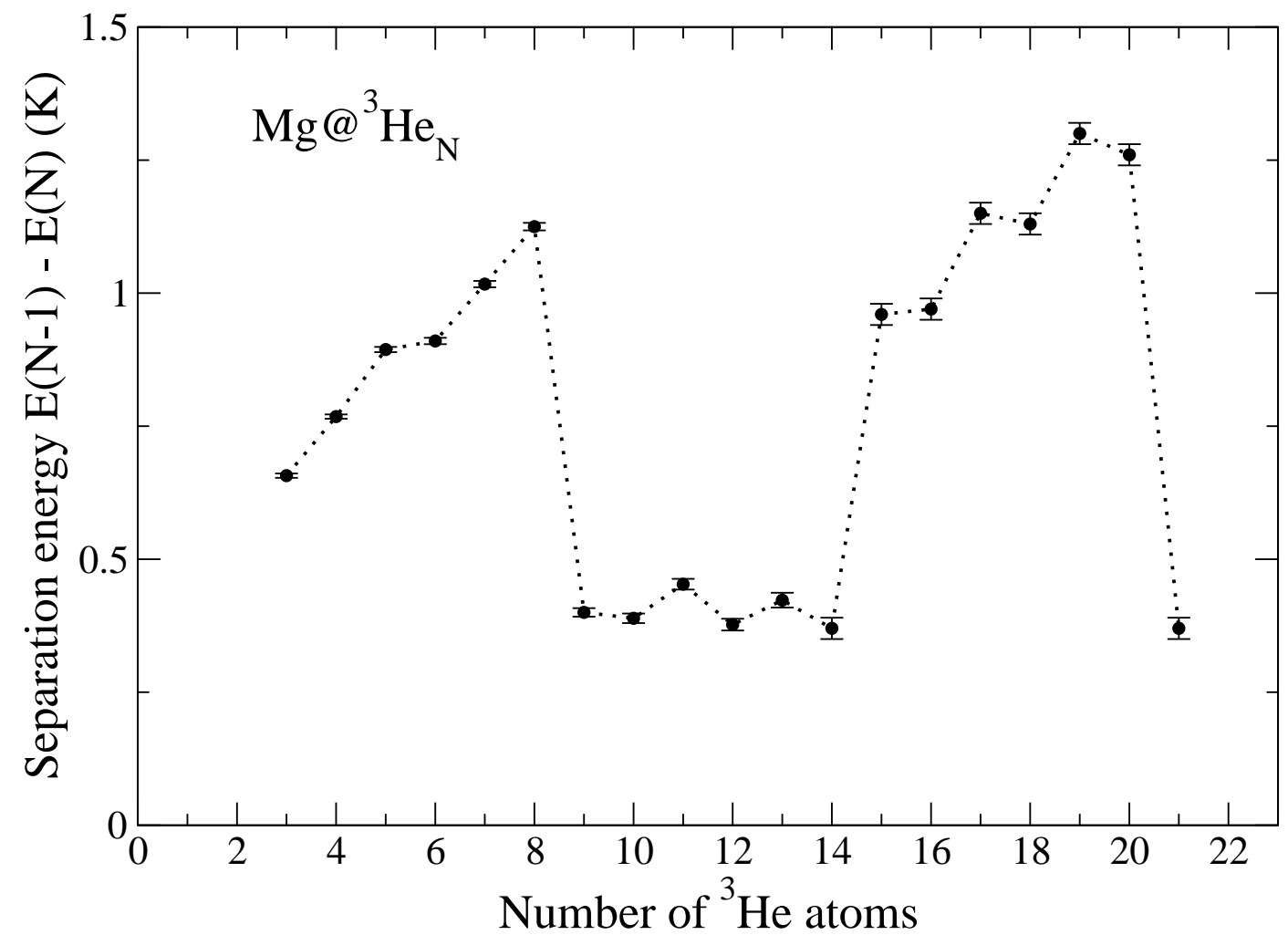

FIG. 10: Separation energy of a ${ }^{3} \mathrm{He}$ atom (in $\mathrm{K}$ ) in $\mathrm{Mg} @{ }^{3} \mathrm{He}_{N_{3}}$ droplets. The dotted line is to guide the eye. 


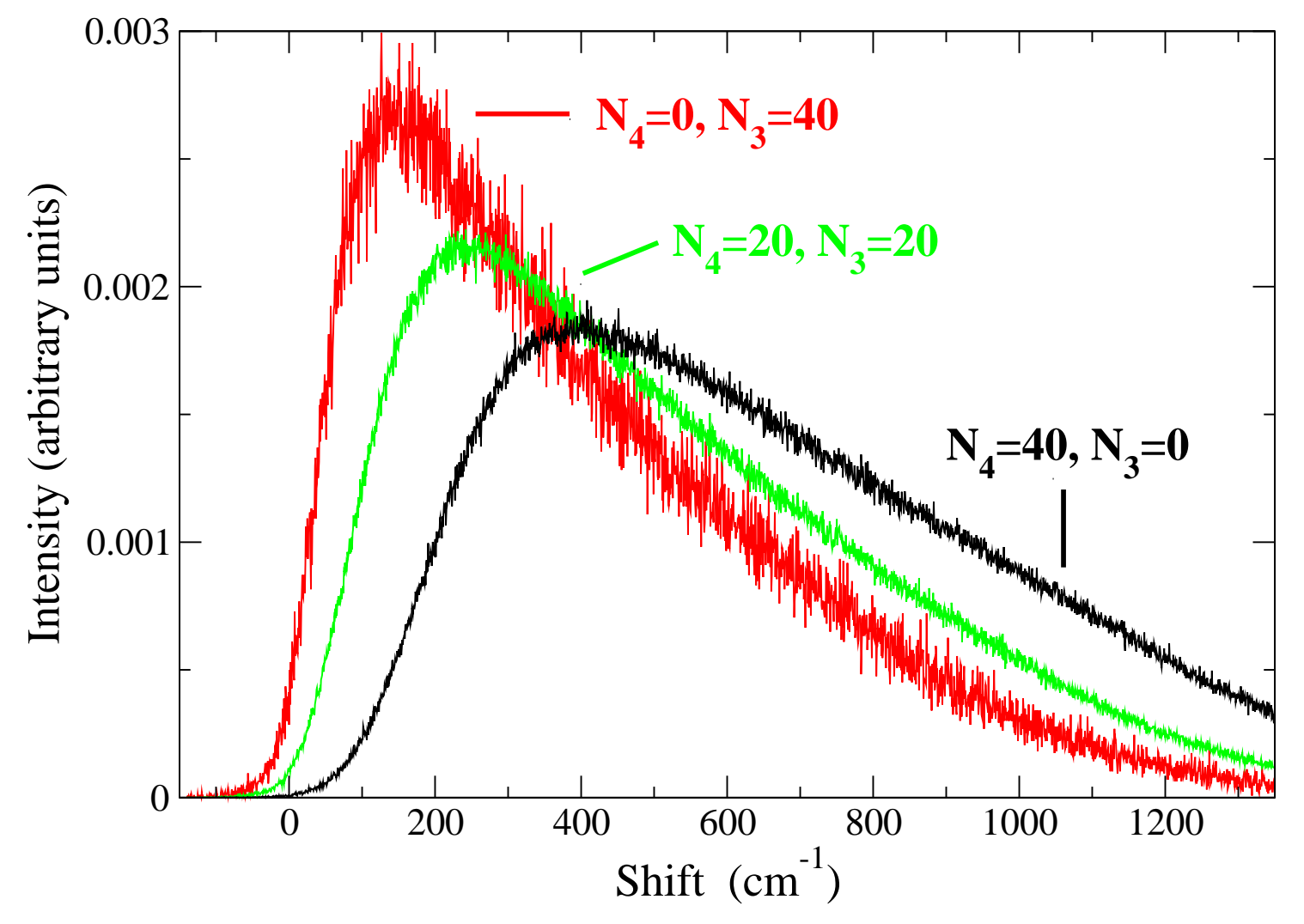

FIG. 11: (Color online) Dipole absorption lines of $\mathrm{Mg}$ in three selected $N_{3}+N_{4}=40$ droplets. 